\title{
Beyond Feeling: Chronic Pain Hurts the Brain, Disrupting the Default-Mode Network Dynamics
}

\author{
Marwan N. Baliki, ${ }^{1}$ Paul Y. Geha, ${ }^{1}$ A. Vania Apkarian, $, 1,2,3,4$ and Dante R. Chialvo ${ }^{1}$ \\ Departments of ${ }^{1}$ Physiology, ${ }^{2}$ Anesthesia, and ${ }^{3}$ Surgery, and ${ }^{4}$ Lurie Cancer Center, Feinberg School of Medicine, Northwestern University, Chicago, \\ Illinois 60611
}

\begin{abstract}
Chronic pain patients suffer from more than just pain; depression and anxiety, sleep disturbances, and decision-making abnormalities (Apkarian et al., 2004a) also significantly diminish their quality of life. Recent studies have demonstrated that chronic pain harms cortical areas unrelated to pain (Apkarian et al., 2004b; Acerra and Moseley, 2005), but whether these structural impairments and behavioral deficits are connected by a single mechanism is as of yet unknown. Here we propose that long-term pain alters the functional connectivity of cortical regions known to be active at rest, i.e., the components of the "default mode network" (DMN). This DMN (Raichle et al., 2001; Greicius et al., 2003; Vincent et al., 2007) is marked by balanced positive and negative correlations between activity in component brain regions. In several disorders, however this balance is disrupted (Fox and Raichle, 2007). Using well validated functional magnetic resonance imaging (fMRI) paradigms to study the DMN (Fox et al., 2005), we investigated whether the impairments of chronic pain patients could be rooted in disturbed DMN dynamics. Studying with fMRI a group of chronic back pain (CBP) patients and healthy controls while executing a simple visual attention task, we discovered that CBP patients, despite performing the task equally well as controls, displayed reduced deactivation in several key DMN regions. These findings demonstrate that chronic pain has a widespread impact on overall brain function, and suggest that disruptions of the DMN may underlie the cognitive and behavioral impairments accompanying chronic pain.
\end{abstract}

Key words: default-mode network; chronic pain; fMRI; resting state networks; functional connectivity; brain

\section{Introduction}

Imaging studies have shown a set of brain regions that usually decrease their activity during task performance (Shulman et al., 1997) when compared with the average brain activity at rest. The fact that these regions were more active at rest than during task performance suggested the existence of a resting state in which the brain remained active in an organized manner, which was called the "default mode" of brain function (Raichle et al., 2001). The regions exhibiting a decrease in activity during task performance are the component members of the "default-mode network" (DMN), which in concerted action maintain the brain resting state (Raichle et al., 2001). Further characterization of the DMN (Raichle et al., 2001; Greicius et al., 2003; Fox et al., 2005; Vincent et al., 2007), either by identifying decreases in brain activity during a task or by the study of correlation patterns of spontaneous brain activity at rest, have provided valuable insight into the intrinsic large-scale functional architecture of the brain (Fox and Raichle, 2007).

Recent studies have already demonstrated that the brain DMN is

Received Sept. 7, 2007; revised Dec. 19, 2007; accepted Dec. 21, 2007.

This work was funded by the National Institutes of Health-National Institute of Neurological Disorders and Stroke. We thank Florencia Chialvo, Philip Hockberger, Seema Khan, Denis Pelli, Oscar Scremin, and Veronika Zsiros for reading this manuscript. We also thank all of the participating patients and volunteers.

Correspondence should be addressed to Dante R. Chialvo, Department of Physiology, Northwestern University, 303 East Chicago Avenue, Chicago, IL 60611. E-mail: d-chialvo@northwestern.edu.

DOI:10.1523/JNEUROSCI.4123-07.2008

Copyright $\odot 2008$ Society for Neuroscience $\quad$ 0270-6474/08/281398-06\$15.00/0 disrupted in autism (Kennedy et al., 2006), Alzheimer' disease (Greicius et al., 2004), depression (Greicius et al., 2007), schizophrenia (Liang et al., 2006; Williamson, 2007), and attention deficit hyperactivity disorder (Tian et al., 2006), suggesting that the study of brain resting activity can be useful to understand disease states as well as potentially provide diagnostic information.

The goal of the present work is to study the integrity of the DMN of a population of chronic pain patients. Pain is considered chronic when it lasts longer than 6 months after the healing of the original injury. Chronic pain is particularly suitable to be studied from this perspective for various reasons. It is known that pain in these patients (at some point) becomes a spontaneous percept, i.e., an intrinsic brain activity occurring even in the absence of explicit brain input or output (Foss et al., 2006). Thus, the alterations in the patient's brain at "rest" can result in a different DMN organization. In turn, potential changes in the DMN activity could be related to symptoms (other than pain) commonly exhibited by chronic pain patients, including depression and anxiety, sleep disturbances, and decision-making abnormalities (Apkarian et al., 2004a), which also significantly diminish their quality of life. The results to be described below show that chronic pain patients display a dramatic alteration in several key DMN regions, suggesting that chronic pain has a widespread impact on overall brain function.

\section{Materials and Methods}

Subjects. Participants were seven female and eight male healthy control subjects (age: $39.6 \pm 3.43$ years; Beck depression index: $8.96 \pm 1.3$; Beck 
anxiety index: $7.46 \pm 1.98)$ and seven female and eight male chronic back pain (CBP) patients [age: $43.8 \pm 4.11$ years; Beck depression index: $7.25 \pm 1.3$; Beck anxiety index: $9.12 \pm 17$; pain duration: $6.3 \pm 0.98$ years $($ mean $\pm \mathrm{SEM})]$. They were all right-handed, and all gave informed consent to procedures approved by Northwestern University Institutional Review Board. Note that there is no significant difference in depression indices between the groups. The patients participated in an earlier study, and their clinical and demographic data, as well as painrelated parameters, have been described (Baliki et al., 2006).

Task. Participants used a finger-spanning device to continuously rate and $\log$ the height of a bar varying in time during fMRI data collection (Baliki et al., 2006). Subjects underwent a training session before scanning, in which they learned to use the finger-span device. The finger span device was synchronized with the fMRI acquisition and connected to a computer. Functional MR data were acquired with a 3T Siemens (Munich, Germany) Trio whole-body scanner with echo planar imaging capability using the standard radio-frequency head coil [scanning parameters were as in Baliki et al. (2006); see supplemental material, available at www.jneurosci.org].

Preprocessing of fMRI data. Blood oxygen level-dependent (BOLD) fMRI time series preprocessing steps included removal of the first four volumes, slice time correction, motion correction using MCFLIRT, spatial smoothing using a Gaussian kernel of full-width-half-maximum 5 $\mathrm{mm}$, linear high-pass temporal filtering, and intensity normalization.

General linear model analysis. To identify significant brain activity BOLD signals were analyzed using FEAT [FMRIB expert analysis tool (Jezzard et al., 2001); www.fmrib.ox.ac.uk/fsl] The fMRI signal was linearly modeled on a voxel-by-voxel basis using FILM (FMRIB's improved linear model) with local autocorrelation correction (Woolrich et al., 2001, 2004), yielding statistical parametric maps for each subject and condition. Average group statistical maps were generated using secondlevel random effects group analysis. A cluster-based correction of the $z$-statistic images was performed and thresholded at $z$ scores $>2.3$. For each resulting cluster of spatially connected voxels surviving the $z$ threshold, a cluster probability threshold of $p=0.01$ was applied to the computed significance of that cluster, which corrects for multiple comparisons (Friston et al., 1995).

Correlation analysis. Correlation maps were produced following the procedures of Fox et al. (2005). In brief, the preprocessed data were bandpass filtered $(0.009<f<0.08 \mathrm{~Hz})$, and motion artifacts were removed through linear regression with the six parameters obtained by rigid body correction of head motion. Correlation maps were constructed by first extracting time series for the seeds (average of a cube of $3 \times 3 \times 3$ voxels centered at the coordinates indicated below) and then computing its correlation coefficient with the time series from all other brain voxels. We examined correlations associated with six predefined seed regions: three regions, referred to as task-positive regions, that exhibited activity increases during our task performance, and three regions, referred to as task-negative regions, that typically exhibited activity decreases (deactivation) during the attention task (Corbetta and Shulman, 2002; Fox et al., 2005). Task-positive regions were centered in the intraparietal sulcus (IPS; $-38,-46,54$ ), the frontal eye field region (FEF; $26,-12,50$ ), and the middle temporal region (MT; $-46,-68,-2$ ). Task-negative regions were centered in the medial prefrontal cortex (mPFC; $-2,46,-16$ ), posterior cingulate/precuneus (PCC; $-4,-50$, $40)$, and lateral parietal cortex (LP; -46, -68, 36).

For each subject and each region, correlation coefficients were converted to normally distributed $z$ scores using the Fischer's transform, i.e., dividing by the square root of the variance, estimated as $1 / \sqrt{(\mathrm{df}-3)}$, where $\mathrm{df}$ corresponds to the degrees of freedom in our measurement (i.e., 240 data points). Because BOLD's consecutive samples are not statistically independent, the degrees of freedom were corrected by a factor of 2.86, in accordance to Bartlett theory (Jenkins and Watts, 1968), resulting in $240 / 2.86=84.5$ degrees of freedom. Finally, group-averaged $z$ scores were computed by combining individual maps using a fixedeffects analysis (Smith et al., 2004). The resulting $t$ values were converted to equally probable $z$ scores and thresholded at significance levels of $p<$ 0.01 .

Conjunction analysis. The group $z$-score maps for the six seed regions were combined by using a conjunction analysis (see Fig. 3) in which voxels were included in the map only if they were significantly correlated or anticorrelated with five of the six seed regions.

Surface-based mapping. Surface-based mapping (see Fig. 3) was constructed using the PALS (population-average, landmark- and surfacebased) average fiducial surface from 12 individual subjects as the atlas target (Van Essen, 2005).

\section{Results}

Results were obtained from experiments as illustrated in Figure 1. The task consisted of tracking the height of a moving bar on a computer screen using a modified joystick attached to one's index finger and thumb. The bar was either quiescent low on the screen (hereafter referred to as "rest") or changing in height with a slow, unpredictable but fixed pattern across subjects (Fig. 1). Subjects' performance was measured by the linear correlation coefficient between the target and tracking time series, and did not differ between the two groups (Fig. 1, top). Despite their similar performance, there were significant differences in brain activity between the groups as demonstrated by the three different methods of analysis described below.

\section{General linear model}

We first used a general linear model to identify the brain regions in which brain BOLD signal changed during the attention task relative to rest. Consistent with extensive earlier work examining visuospatial attention tasks (Vandenberghe et al., 2001; Corbetta and Shulman, 2002; Pihlajamäki et al., 2005), dominant activations were located in posterior parietal and lateral prefrontal cortices, whereas deactivations occurred mainly within $\mathrm{mPFC}$ and PCC. Although activations in CBP patients' and controls' brains were similar, CBP patients exhibited significantly less deactivations than healthy subjects in mPFC, amygdala, and PCC (Fig. 1; supplemental Table 1, available at www.jneurosci.org as supplemental material). Potential artifacts that might contribute to such a difference were investigated and discarded [i.e., different degrees of compliance, and nonstationarity of task performance (see supplemental material, available at www.jneurosci.org)]. Further analysis was directed to identify the origin of these differences as described in the next paragraphs.

\section{Task-triggered BOLD average}

The main group differences shown in Figure 1 are linked to deactivation with respect to the attention task; thus, we specifically investigated the phase relation between the BOLD signals and the task. Figure 2 shows the results for two relevant regions. One is the left intraparietal sulcus (LIPS), which is activated during the task, and the other the mPFC, which is deactivated by the task. Simple inspection of the time courses of LIPS and mPFC activity (Fig. 2a) confirms that they move in opposite directions with respect to the task. Nevertheless, quantitative analysis in both groups reveals important differences. Although mPFC activity in both groups was negatively correlated with the task, the magnitude value of the correlation was significantly attenuated in CBP patients. This is quantified by the average BOLD responses associated with the rest-to-active phase task transition, i.e., the tasktriggered BOLD average (Fig. $2 b$ ), which demonstrates a significant deactivation failure in the $\mathrm{mPFC}$ of $\mathrm{CBP}$ patients. Meanwhile there were no significant group differences in both LIPS activity and magnitude tracking. Further calculations, as shown in the next section, reveal the spatial extent of these similarities and differences. 


\section{Seed correlation maps}

To characterize the full impact of the CBPassociated changes in BOLD activationdeactivation, we used recently described methods (Fox et al., 2005) (see supplemental material, available at www. jneurosci.org). Briefly, a given region was used as a seed to compute its linear correlation coefficients against all other brain voxels' time series (Fig. 3). The resultant correlation maps summarize the functional coactivation between the seed region with the rest of the cortex. Three of the seeds (mPFC, PCC, and LP) are known to be deactivated during an attention task ("task-negative") and three others (IPS, $\mathrm{FEF}$, and MT) are activated during the task ("task-positive").

The correlation maps that we obtained from the control group (Fig. 3, left) replicate very closely the DMN described at rest (Raichle et al., 2001; Fox et al., 2005; Raichle, 2006; Mason et al., 2007), a finding consistent with observations already showing that in minimally demanding tasks brain functional connectivity approximates the functional connectivity seen during rest (Greicius et al., 2003, 2004; Fox et al., 2005). A dramatic difference is immediately apparent in the patients' data. The maps computed from the CBP patients' data (Fig. 3, right) show much smaller cortical domains with negative correlations, and larger domains with positive correlations compared with controls. In the bottom plots, the conjunction maps provide a summary of the consistency of these correlations across all seeds. To provide a quantitative estimation of these alterations, we calculated the ratio between the area (in number of voxels) correlated versus anticorrelated for each of the six seeds (Fig. 4). Whereas healthy subjects maintain a ratio close to unity, CBP patients exhibit ratios up to thirty times larger.

The reduction in the total area exhibiting anticorrelation (Fig. 3) is consistent with the results presented in Figure 1, as well as with the calculations presented in Figure 2. Thus, the three methods provide consistent evidence of a disruption in the cortical functional connectivity in CBP patients, with impaired deactivation of the DMN.

In addition, we evaluated the relationship between each patient's fMRI activity with his or her pain characteristics (e.g., intensity, duration, depression, and anxiety), and we found that the extent of mPFC deactivation correlated with the number of years of pain suffering endured $(r=0.45 ; p=0.09)$.

\section{Discussion}

These experiments were designed to search in CBP patients for potential differences in their brain resting activity as reflected by the default mode network. Hence, the focus was on identifying differences in the way CBP patients' brains process information not related to pain. This was motivated by the hypothesis that unrelenting pain could leave a mark beyond cortical circuits involved in perception. Thus, from this viewpoint, using an attention task to infer something relevant about the status of pain in CBP patients' brains is fully justified.

The approach proved very prolific. This is the first study demonstrating that CBP patients exhibit severe alterations in the functional connectivity between brain regions implicated in the default mode network. It seems that enduring pain for a long time affects brain function in response to even minimally demanding attention tasks completely unrelated to pain. Furthermore, the fact that the observed task performance, compared with healthy 


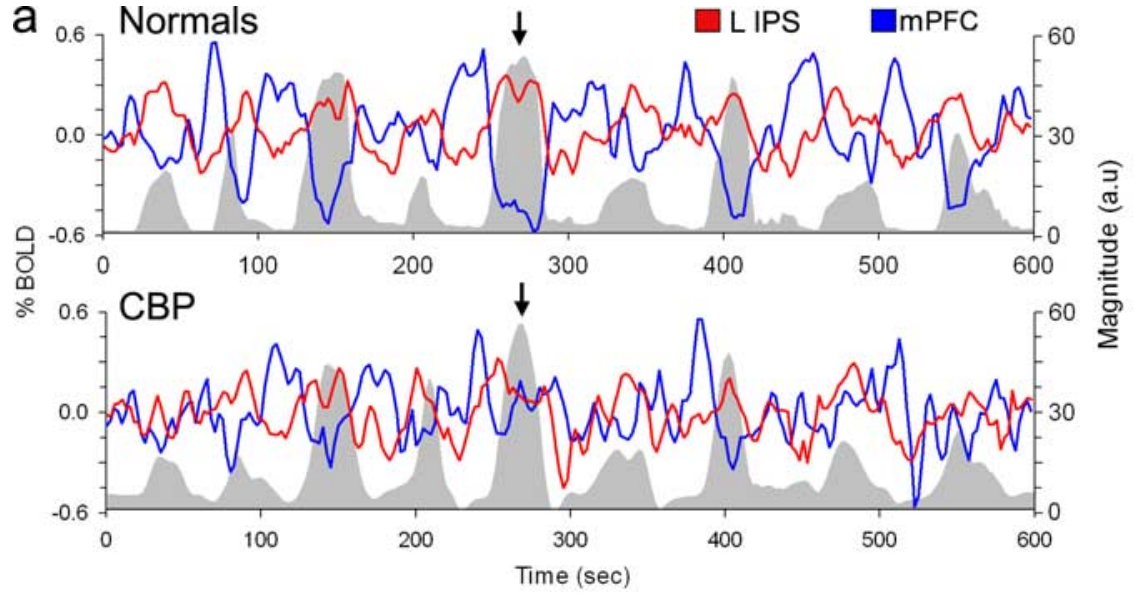

b
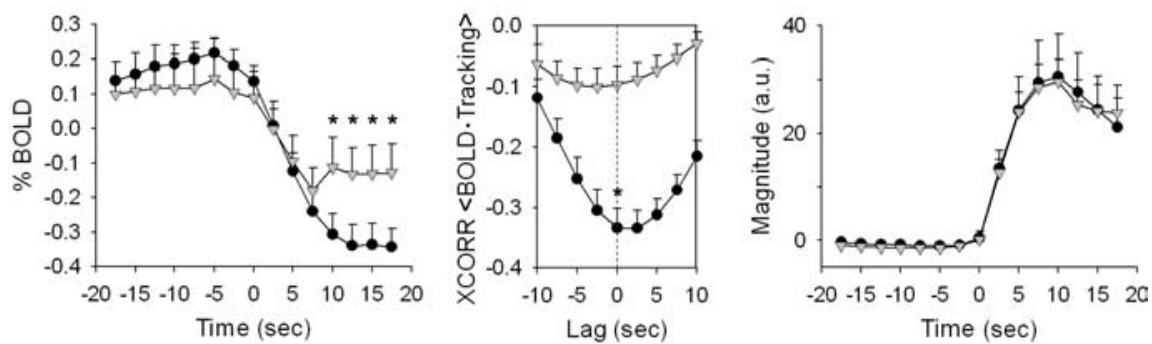

\section{IPS}
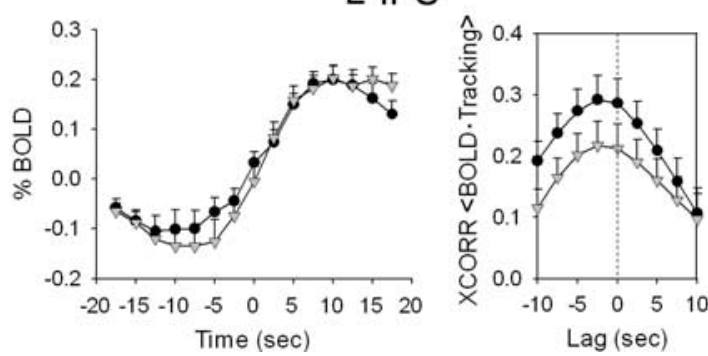

Figure 2. Differences in time course of BOLD signal between CBP patients and healthy controls. a, Group-averaged BOLD signals from mPFC (blue) and LIPS (red) for controls (top) and CBP patients (bottom) are shown superimposed on the respective group-averaged tracking time courses (gray). The main result illustrated here is that mPFC BOLD signal is more deactivated in normal subjects than in CBP patients each time the subject engages in tracking. The arrow indicates a time in which these differences can be appreciated even by simple inspection of the traces. $\boldsymbol{b}$, Time course of average BOLD responses for mPFC (top) and LIPS (bottom) relative to transition from rest to tracking. Task-triggered BOLD signals averaged over 135 tracking events were significantly smaller in mPFC of CBP patients selectively in the deactivation phase ( $\left.{ }^{*} p<0.01\right)$, at times of peak tracking ( $10-20$ s from start of tracking), whereas task performance (magnitude tracking) did not differ between the two groups (top right). The LIPS responses were similar between the groups. Middle, Cross-correlations between BOLD signal and tracking time course for each group for different time lags ( -10 to $10 \mathrm{~s}$ ) revealed that the $\mathrm{mPFC}$ BOLD signal was anticorrelated to the task time course in normal subjects (mean $r=-0.35 \pm 0.2, \mathrm{SEM}$, at lag $=2.5 \mathrm{~s}$ ), and that this anticorrelation was significantly attenuated (mean $r=-0.11 \pm 0.20$, SEM, at lag $=-2.5 s ; p<0.001)$ in (BP patients. LIPS signal was positively correlated to task execution and did not differ between the groups.

subjects, is unaffected, whereas the brain activity is dramatically different, raises the question of how other behaviors are impaired by the altered brain activity.

Given that CBP patients report a fluctuating but relentless pain percept (Foss et al., 2006) rendering their brain never truly at rest, it is reasonable to expect them to have an altered brain resting state as described here. It has been proposed that in the normal brain, the DMN provides "a balance of opposing forces" (Raichle, 2006) to enhance "the maintenance of information for interpreting, responding to, and even predicting environmental demands" (Raichle, 2006). Therefore, the "unbalanced" DMN that we observe in CBP patients suggests that these functions may be compromised, something that deserves further studies. Data collected in CBP patients during resting state should exhibit similar disruption of the correlated-anticorrelated maps, given the reported (Fox and Raichle, 2007) correspondence between DMN and resting state fluctuations.

By design, the present study cannot provide mechanistic explanations. However, the disruption of functional connectivity observed here with increased CBP duration may be related to the earlier observation of brain atrophy increasing with pain duration also in CBP patients (Apkarian et al., 2004b). CBP patients exhibit increased mPFC activity in relation to spontaneous pain (Baliki et al., 2006), in addition to dorsolateral prefrontal cortex (dlPFC) atrophy. Therefore, the decreased deactivations described here may be related to the dlPFC/mPFC mutual inhibitory interactions perturbed with time. If that is the case, it will support the idea of a plastic, time-dependent, reorganization of the brain as patients continue to suffer from CBP. Mechanistically, the early stages of this cortical reorganization may be driven by peripheral and spinal cord events, such as those that have been documented in animal models of chronic pain (Woolf and Salter, 2000; Julius and Basbaum, 2001), whereas later events may be related to coping strategies necessary for living with unrelenting pain. It is important to recognize that transient but repetitive functional alterations can lead to more permanent changes. Accordingly, longterm interference with normal DMN activity may eventually initiate plastic changes that could alter irreversibly the stability and subsequently the conformation of the resting state networks. Elucidation of the interaction between such mechanisms with brain atrophy and with peripheral and spinal cord reorganization will require future studies.

How do the disrupted correlation-anticorrelation patterns relate to patterns of neural activity? Our current understanding is clearly insufficient to answer this question, and further studies are needed, but we can speculate that, up to a certain degree, increases in positive correlations could be interpreted as relative increases in neuronal synchrony and/or activity. Conversely, deactivated regions could be seen as a result of increased inhibitory drive or asynchronous neuronal firing. These relations, however, cannot be easily treated in isolation or dissected, because they emerge from mutual interactions among all the members of the DMN. Finally, an additional layer of complexity is added to these interactions because they seem to be frequency dependent, as suggested by recent modeling (Honey et al., 2007) and experimental (Mantini et al., 2007) results. 

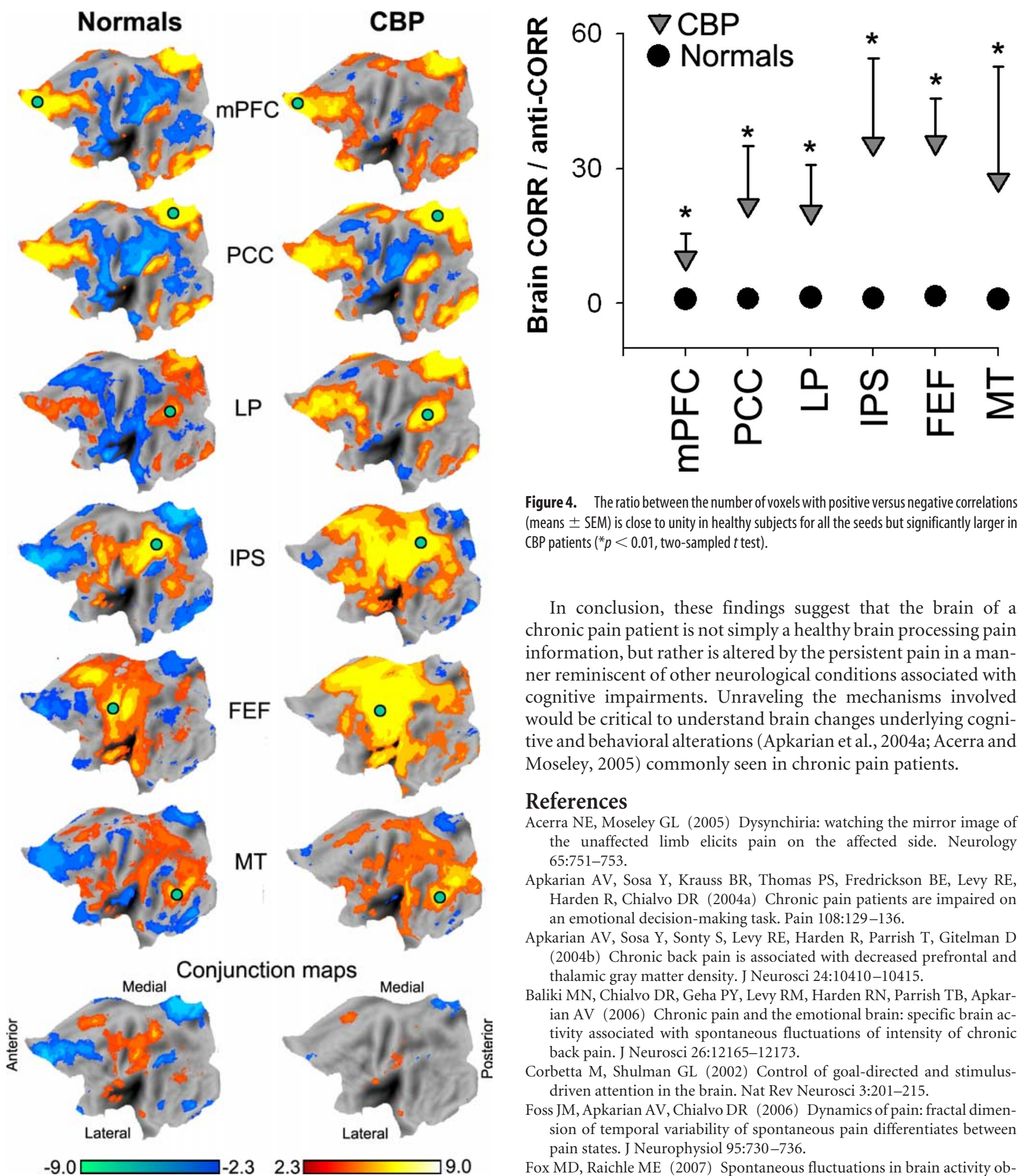

Figure 3. Disrupted correlation maps in CBP patients. Group-averaged z-score maps $(n=15$ in each group) showing regions with significant correlations with the six seed regions (small circles) in normal controls (left) and in CBP patients (right). Results are shown for the three task-negative seed regions ( $\mathrm{mPFC}, \mathrm{PCC}$, and $\mathrm{LP}$ ) and three task-positive seed regions (IPS, FEF, and MT). Regions with positive correlations (red-yellow) have $z$ scores $>2.3(p<0.01)$, and those with negative correlations (blue-green) havezscores less than $-2.3(p<0.01)$. Notice that in the CBP patients' map, the majority of regions identified in the control subjects as being negatively correlated (i.e., blue colored) are missing.

Figure 4. The ratio between the number of voxels with positive versus negative correlations (means \pm SEM) is close to unity in healthy subjects for all the seeds but significantly larger in CBP patients $\left({ }^{*} p<0.01\right.$, two-sampled $t$ test).

In conclusion, these findings suggest that the brain of a chronic pain patient is not simply a healthy brain processing pain information, but rather is altered by the persistent pain in a manner reminiscent of other neurological conditions associated with cognitive impairments. Unraveling the mechanisms involved would be critical to understand brain changes underlying cognitive and behavioral alterations (Apkarian et al., 2004a; Acerra and Moseley, 2005) commonly seen in chronic pain patients.

\section{References}

Acerra NE, Moseley GL (2005) Dysynchiria: watching the mirror image of the unaffected limb elicits pain on the affected side. Neurology 65:751-753.

Apkarian AV, Sosa Y, Krauss BR, Thomas PS, Fredrickson BE, Levy RE, Harden R, Chialvo DR (2004a) Chronic pain patients are impaired on an emotional decision-making task. Pain 108:129-136.

Apkarian AV, Sosa Y, Sonty S, Levy RE, Harden R, Parrish T, Gitelman D (2004b) Chronic back pain is associated with decreased prefrontal and thalamic gray matter density. J Neurosci 24:10410-10415.

Baliki MN, Chialvo DR, Geha PY, Levy RM, Harden RN, Parrish TB, Apkarian AV (2006) Chronic pain and the emotional brain: specific brain activity associated with spontaneous fluctuations of intensity of chronic back pain. J Neurosci 26:12165-12173.

Corbetta M, Shulman GL (2002) Control of goal-directed and stimulusdriven attention in the brain. Nat Rev Neurosci 3:201-215.

Foss JM, Apkarian AV, Chialvo DR (2006) Dynamics of pain: fractal dimension of temporal variability of spontaneous pain differentiates between pain states. J Neurophysiol 95:730-736.

Fox MD, Raichle ME (2007) Spontaneous fluctuations in brain activity observed with functional magnetic resonance imaging. Nat Rev Neurosci 8:701-711.

Fox MD, Snyder AZ, Vincent JL, Corbetta M, Van Essen DC, Raichle ME (2005) The human brain is intrinsically organized into dynamic, anticorrelated functional networks. Proc Natl Acad Sci USA 102:9673-9678.

Friston KJ, Holmes AP, Worsley KJ, Poline J-B, Frith CD, Frackowiak RS (1995) Statistic parametric maps in functional imaging: a general linear approach. Hum Brain Mapp 2:189-210.

Greicius MD, Krasnow B, Reiss AL, Menon V (2003) Functional connectivity in the resting brain: a network analysis of the default mode hypothesis. Proc Natl Acad Sci USA 100:253-258.

Greicius MD, Srivastava G, Reiss AL, Menon V (2004) Default-mode net- 
work activity distinguishes Alzheimer's disease from healthy aging: evidence from functional MRI. Proc Natl Acad Sci USA 101:4637-4642.

Greicius MD, Flores BH, Menon V, Glover GH, Solvason HB, Kenna H, Reiss AL, Schatzberg AF (2007) Resting-state functional connectivity in major depression: abnormally increased contributions from subgenual cingulate cortex and thalamus. Biol Psychiatry 62:429-437.

Honey CJ, Kotter R, Breakspear M, Sporns O (2007) Network structure of cerebral cortex shapes functional connectivity on multiple time scales. Proc Natl Acad Sci USA 104:10240-10245.

Jenkins GM, Watts DG (1968) Spectral analysis and its applications. Boca Raton, FL: Emerson-Adams.

Jezzard P, Mathews P, Smith SM (2001) Functional MRI: an introduction to methods. Oxford: Oxford UP.

Julius D, Basbaum AI (2001) Molecular mechanisms of nociception. Nature 413:203-210.

Kennedy DP, Redcay E, Courchesne E (2006) Failing to deactivate: resting functional abnormalities in autism. Proc Natl Acad Sci USA 103:8275-8280.

Liang M, Zhou Y, Jiang T, Liu Z, Tian L, Liu H, Hao Y (2006) Widespread functional disconnectivity in schizophrenia with resting-state functional magnetic resonance imaging. NeuroReport 17:209-213.

Mantini D, Perrucci MG, Del Gratta C, Romani GL, Corbetta M (2007) Electrophysiological signatures of resting state networks in the human brain. Proc Natl Acad Sci USA 104:13170-13175.

Mason MF, Norton MI, Van Horn JD, Wegner DM, Grafton ST, Macrae CN (2007) Wandering minds: the default network and stimulusindependent thought. Science 315:393-395.

Pihlajamäki M, Tanila H, Könönen M, Hänninen T, Aronen HJ, Soininen H (2005) Distinct and overlapping fMRI activation networks for processing of novel identities and locations of objects. Eur J Neurosci 22:2095-2105.

Raichle ME (2006) Neuroscience. The brain's dark energy. Science 314:1249-1250.

Raichle ME, MacLeod AM, Snyder AZ, Powers WJ, Gusnard DA, Shulman
GL (2001) A default mode of brain function. Proc Natl Acad Sci USA 98:676-682.

Shulman GL, Fiez J, Corbetta M, Buckner R, Miezin FM, Raichle ME, Petersen S (1997) Common blood flow changes across visual task: II Decreases in cerebral cortex. J Cogn Neurosci 9:648-663.

Smith SM, Jenkinson M, Woolrich MW, Beckmann CF, Behrens TE, Johansen-Berg H, Bannister P, De Luca CJ, Drobnjak I, Flitney DE, Nianzy R, Saunders J, Vickers J, Zhang Y, De Stefano N, Brady JM, Mathews PM (2004) Advances in functional and structural MR image analysis and implementation as FSL. NeuroImage 23 [Suppl 1]:S208-S219.

Tian L, Jiang T, Wang Y, Zang Y, He Y, Liang M, Sui M, Cao Q, Hu S, Peng M, Zhuo Y (2006) Altered resting-state functional connectivity patterns of anterior cingulate cortex in adolescents with attention deficit hyperactivity disorder. Neurosci Lett 400:39-43.

Van Essen DC (2005) A population-average, landmark- and surface-based (PALS) atlas of human cerebral cortex. NeuroImage 28:635-662.

Vandenberghe R, Gitelman DR, Parrish TB, Mesulam MM (2001) Location- or feature-based targeting of peripheral attention. NeuroImage 14:37-47.

Vincent JL, Patel GH, Fox MD, Snyder AZ, Baker JT, Van Essen DC, Zempel JM, Snyder LH, Corbetta M, Raichle ME (2007) Intrinsic functional architecture in the anaesthetized monkey brain. Nature 447:83-85.

Williamson P (2007) Are anticorrelated networks in the brain relevant to schizophrenia? Schizophr Bull 33:994-1003.

Woolf CJ, Salter MW (2000) Neuronal plasticity: increasing the gain in pain. Science 288:1765-1769.

Woolrich MW, Ripley BD, Brady M, Smith SM (2001) Temporal autocorrelation in univariate linear modeling of FMRI data. NeuroImage 14: $1370-1386$.

Woolrich MW, Behrens TE, Beckmann CF, Jenkinson M, Smith SM (2004) Multilevel linear modelling for FMRI group analysis using Bayesian inference. NeuroImage 21:1732-1747. 